\title{
NÍVEIS DE ESTRESSE OXIDATIVO AO DIAGNÓSTICO E SUA ASSOCIAÇÃO COM O PERFIL DE SOBREVIDA EM PACIENTES PORTADORAS DE CÂNCER DE MAMA OPERÁVEIS EM ESTADIOS INICIAIS: UM ESTUDO PILOTO
}

\author{
LEVELS OF OXIDATIVE STRESS TO DIAGNOSIS AND THEIR \\ ASSOCIATION WITH THE SURVIVAL PROFILE IN PATIENTS WITH \\ BREAST CANCER OPERABLE IN INITIAL STAGES: A PILOT STUDY
}

\author{
Souza, LSB ${ }^{1,3}$ Rech, D ${ }^{2,3}$, Bufalo Kawassaki, AC ${ }^{2,3}$ Calistro da Silva, DFH ${ }^{1}$ Malanowski, $\mathbf{J}^{1,3}$ \\ Santos, HJ ${ }^{1,3}$ Alves, FM ${ }^{1,3}$ Maito, VT ${ }^{1,3}$ Campestrini, BV ${ }^{1,3}$ Oliveira, ST ${ }^{1,3}$ Panis, C 2,3,4 \\ ${ }^{1}$ Discente do curso de Medicina da Universidade Estadual do Oeste do Paraná, Francisco Beltrão - PR, Brasil. \\ 2 Docente do curso de Medicina da Universidade Estadual do Oeste do Paraná, Francisco Beltrão - PR, Brasil. \\ ${ }^{3}$ Laboratório de Biologia de Tumores, Universidade Estadual do Oeste do Paraná, Francisco Beltrão, Brasil. \\ 4 Programa de Pós-Graduação em Ciências Aplicadas à Saúde, Unioeste, Francisco Beltrão-PR, Brasil. \\ Endereço do autor de correspondência: Carolina Panis. Universidade Estadual do Oeste do Paraná, Centro de Ciências da Saúde. Rodovia \\ Vitorio Traiano km 2, Francisco Beltrão_PR. carolpanis@hotmail.com
}

\section{Resumo}

\begin{abstract}
Altos níveis de estresse oxidativo têm sido associados a pior prognóstico do câncer de mama, mas não se sabe sua relação como possível preditor de sobrevida. Neste estudo investigamos a associação entre os níveis circulantes de estresse oxidativo no momento do diagnóstico e o perfil de sobrevida de pacientes portadoras de tumores de mama operáveis. Foi realizado um estudo prospectivo em uma população de 606 pacientes do sexo feminino com tumores de mama em estadios iniciais entre os anos de 2015-2020. Dentre essas pacientes, 18 vieram a óbito no seguimento do estudo, sendo pareadas com 36 pacientes que permaneceram vivas no período do estudo. Amostras de sangue foram coletadas ao diagnóstico, e os níveis de estresse quantificados por meio da dosagem dos metabólitos do óxido nítrico (NO) e perfil de peroxidação lipídica plasmática. No grupo óbito, observou-se perfil de lipoperoxidação plasmática e níveis de NO elevados ao diagnóstico, quando comparado ao grupo de pacientes vivas. Altos níveis de lipoperoxidação plasmática foram diretamente relacionados com o desenvolvimento de metástases no grupo de pacientes vivas. Os resultados sugerem que o perfil oxidativo sistêmico aumentado ao diagnóstico observado em portadoras de câncer de mama está diretamente relacionado ao desfecho óbito em 5 anos.
\end{abstract}

Palavras-chave: estresse oxidativo; câncer de mama; mortalidade.

\section{Abstract}

High levels of oxidative stress have been associated with a worse prognosis of breast cancer, but its relationship is not known as a possible predictor of survival. In this study we investigated the association between circulating levels of oxidative stress at the time of diagnosis and the survival profile of patients with operable breast tumors. A prospective study was conducted in a population of 606 female patients with early stage breast tumors between the years 2015-2020. Among these patients, 18 died during the study, being paired with 36 patients who remained alive during the study period. Blood samples were collected at diagnosis, and stress levels were quantified by measuring the nitric oxide (NO) metabolites and plasma lipid peroxidation profile. In the death group, a plasma lipoperoxidation profile and high NO levels at diagnosis were observed when compared to the group of living patients. High levels of plasma lipoperoxidation were directly related to the development of metastases in the group of living patients. The results suggest that the increased systemic oxidative profile at the diagnosis observed in patients with breast cancer is directly related to the outcome of death in 5 years.

Keywords: oxidative stress; breast cancer; mortality. 


\section{Introdução}

Durante a respiração celular, a atividade metabólica mitocondrial gera espécies reativas de oxigênio (EROs). No entanto, devido a uma ampla gama de sensores redox, tais substâncias são equilibradas por agentes antioxidantes, de forma a manter o status redox da célula. Em alguns casos, ocorre um desequilíbrio desse mecanismo, seja por uma geração excessiva de EROs ou por uma falha nos sensores redox, fazendo com que ocorra um desbalanço na relação oxidação-redução celular, o que é chamado de estresse oxidativo ${ }^{1}$.

O estresso oxidativo está implicado na base de diversas patologias conhecidas, dentre elas o câncer de mama ${ }^{1,2}$. Sabe-se que os tumores de mama estão naturalmente incorporados a um ambiente pró-oxidante: o tecido adiposo ${ }^{3}$. Nesse tecido, EROs são amplamente produzidas, principalmente por meio do óxido nítrico (NO), e o seu excedente atua rapidamente na vizinhança lipídica, desregulando funções celulares essenciais, como a replicação e reparo do DNA ${ }^{4,5}$, e produzindo metabólitos ativos que participam de fenômenos centrais na progressão tumoral, como a angiogênese e a vasodilatação ${ }^{5,6}$. Além disso, esse ambiente pró- oxidante parece afetar a adaptação das células tumorais contra o efeito gerado por drogas antineoplásicas, comprometendo a eficácia da quimioterapia $^{7}$.

Estudos têm apontado que há uma relação direta entre um excedente de EROs e parâmetros determinantes no prognóstico do câncer de mama. Estudo publicado por Pires e colaboradores caracterizou o trinômio estresse oxidativo, inflamação e sistema complemento como sendo um eixo central para a quimiorresistência no subtipo de câncer de mama luminal A, em especial no tratamento combinado de paclitaxel e doxorrubicina ${ }^{8}$. Sugere-se que o remanescente da produção de radicais livres possa favorecer a interação dessas moléculas com o DNA, produzindo modificação de bases, induzindo crosslinking inter e intracadeia ou promovendo ligações cruzadas que levam à carcinogênese ${ }^{9}$. Assim, este contexto proporciona um microambiente que favorece o aparecimento de metástases à distância, fenômeno considerado com a principal causa de morte em pacientes com câncer.

A relação entre estresse oxidativo e os aspectos clinicopatológicos do câncer de mama mostra que a presença do tumor primário é 
indicativa de um estado pró-oxidante sistêmico no câncer de mama e altos níveis de peroxidação lipídica estão associados à recorrência da doença ${ }^{10}$. Além disso, sabe-se que a quimioterapia modula a homeostase redox sistêmica em mulheres com câncer de mama ${ }^{11,12}$. Adicionalmente, a literatura sugere que o aumento do estresse oxidativo e o comprometimento da resposta imune podem ser necessários para garantir a progressão do câncer para estágios avançados e podem resultar de mediadores inflamatórios do hospedeiro e do tumor $^{13}$. Entretanto, não está claro qual a relação entre o perfil redox sistêmico e a ocorrência de óbitos em pacientes portadoras de câncer de mama.

Apesar de diversos estudos investigarem as alterações decorrentes de EROs no cenário do câncer de mama, não há na literatura científica clareza sobre o uso destes parâmetros como possíveis marcadores preditores de sobrevida. Nesse contexto, o objetivo deste estudo foi investigar se há correlação entre o perfil de estresse oxidativo sistêmico ao diagnóstico com o perfil clínico de sobrevida de mulheres portadoras de câncer de mama operável.
Métodos

\section{Desenho do estudo}

Esta pesquisa caracteriza-se como um estudo prospectivo em que foram analisadas 606 pacientes do sexo feminino com tumores de mama em estadios iniciais que compareceram a um hospital público oncológico do interior do Paraná, Brasil, entre os períodos de 2015 e 2020. Dentre essas pacientes, 18 vieram a óbito no seguimento do estudo devido a causas relacionadas ao câncer de mama. Cada paciente que veio a óbito foi pareada com outras duas pacientes vivas estudadas. Para selecionar tais pacientes, as variáveis utilizadas foram idade ao diagnóstico igual ou \pm 2 anos e presença de câncer de mama, a fim de que os grupos denominados "grupo óbito" $(\mathrm{n}=18)$ e "grupo vivo" $(\mathrm{n}=36)$ fossem o mais semelhante possível. Dados clínicos e histopatológicos foram obtidos por meio da análise de prontuários. Este estudo foi aprovado pelo Comitê de Ética em Pesquisa da Instituição e pelo Conselho Nacional de Ética em Pesquisa (CAAE número 35524814.4.0000.0107). Todas as participantes assinaram o termo de consentimento livre e esclarecido. 


\section{Coleta das amostras}

Foram obtidas amostras de sangue (10 mL) das pacientes ao diagnóstico, antes de intervenções terapêuticas relacionadas ao câncer de mama, por punção venosa periférica e armazenadas em tubos com EDTA. Os tubos foram centrifugados durante 5 minutos a 4000 rpm, e o plasma resultante transferido, com o auxílio de uma micropipeta, para tubos de criopreservação armazenados em freezer a $-20^{\circ}$ C para posterior análise.

\section{Determinação do perfil de estresse oxidativo}

O status oxidativo das amostras foi determinado pela medida do conteúdo total de metabólitos do óxido nítrico (NO) ${ }^{14}$ e perfil de peroxidação lipídica plasmática medida por quimioluminescência ${ }^{15}$. Para o NO, alíquotas de $60 \mu \mathrm{L}$ de células contendo sobrenadante $(5 \times 105$ células / mL) foram desprotenizadas por adição de $50 \mu \mathrm{L}$ de $\mathrm{ZnSO} 4(75 \mathrm{mM})$, centrifugadas a $10000 \mathrm{rpm}$ por 2 minutos e depois misturadas com $70 \mu \mathrm{L}$ de $\mathrm{NaOH} \quad(55 \mathrm{mM})$. Após centrifugação de $10000 \mathrm{rpm}$ por 5 minutos, foram adicionados $150 \mu \mathrm{L}$ de sobrenadante a 50 $\mu \mathrm{L}$ de tampão de glicina $(45 \mathrm{~g} / \mathrm{L}, \mathrm{pH} 9,7) \mathrm{e}$ incubados por 10 minutos com grânulos de cádmio previamente ativados por $\mathrm{CuSO} 4$ ( $5 \mathrm{mM}$, 5 minutos). Depois, alíquotas de $50 \mu \mathrm{L}$ foram misturadas com o reagente de Griess por 10 minutos, e a absorbância foi lida a $550 \mathrm{~nm}$. Os resultados foram expressos como $\mathrm{uM}$ de nitrito. O perfil de peroxidação lipídica foi obtido misturando $500 \mu \mathrm{L}$ de amostra, $500 \mu \mathrm{L}$ de tampão fosfato $\left(\mathrm{K}_{2} \mathrm{HPO}_{4} 30 \mathrm{mM}\right.$ em KCl $1,15 \%$, $\mathrm{pH} 7,4,370 \mathrm{C})$ e $20 \mu \mathrm{L}$ de hidroperóxido de tertbutil $(3 \mathrm{mM})$. Os dados foram obtidos em um luminômetro Glomax (Promega) e os resultados analisados no software OriginLab 7.5. Os dados foram expressos como unidades de luz relativa (URL).

\section{Análise estatística}

As análises estatísticas foram realizadas usando o software GraphPad Prism 7.0, Microsoft Office Excel 2016 e OriginLab 9.0. As diferenças entre os grupos foram avaliadas pela análise de variância bidirecional (ANOVA) com o teste post hoc de Bonferroni para as curvas de peroxidação lipídica e pelo teste $t$ de Student ou teste de Mann-Whitney dependendo do número de grupos em comparação. Correlações entre parâmetros no 
plasma e tecido tumoral também foram realizadas usando os testes de Pearson ou Spearman. Um valor de $p<0,05$ foi adotado como significante.

\section{Resultados}

A análise dos dados clinicopatológicos

(tabela 1) revelou que no grupo vivo a positividade para os receptores de estrogênio e progesterona foi maior $(80,6 \%)$ quando comparado ao grupo óbito $(41,2 \%)$. Com relação ao tamanho do tumor, ambos os grupos se apresentaram majoritariamente com tumores menores que $5 \mathrm{~cm}$, sendo $41,2 \%$ dos tumores menores que $2 \mathrm{~cm}$ no grupo óbito e $40 \%$ no grupo vivo. $\mathrm{O}$ valor do marcador $\mathrm{Ki}-67 \geq 14 \%$ foi levemente maior nas pacientes que foram a óbito no seguimento do estudo $(68,7 \%)$ em relação às pacientes vivas $(66,6 \%)$. Da mesma forma, o subgrupo óbito apresentou maior percentual de metástase linfonodal (60\%) e presença de êmbolos angiolinfáticos $(66,7 \%)$ quando comparados ao subgrupo vivo. A avaliação histopatológica evidenciou que no grupo vivo o percentual de pacientes com grau histológico I, II e III foi de $44,4 \%, 41,7 \%$ e $13,9 \%$, respectivamente. No grupo óbito metade das pacientes enquadravam-se no grau histológico II, enquanto $18,7 \%$ no grau I e $31,3 \%$ no grau III.
Com relação ao Índice de Massa Corpórea (IMC), o grupo óbito apresentou maior percentual de pacientes obesas $(22,2 \%)$ quando comparado ao grupo vivo $(17,2 \%)$, entretanto o grupo vivo apresentou mais pacientes com sobrepeso $(45,7 \%)$ contraposto ao grupo óbito $(38,9 \%)$. Ambos os grupos apresentaram o mesmo percentual de pacientes com menopausa ao diagnóstico $(72,2 \%)$ e idade > 50 anos (72,2\%), com média de $52 \pm 8$ anos no grupo óbito e $56 \pm 8$ anos no vivo.

A figura 1 evidencia o perfil de peroxidação plasmática determinado por quimiluminescência de alta sensibilidade, onde obtivemos valores mais elevados nas pacientes que foram a óbito comparado às pacientes vivas (Mín 727057 - Máx 3005765, Intervalo de confiança 95\% 1189636 - 223843 para o grupo óbito e Mín 440457 - Máx 1477520, Intervalo de confiança 95\% 604890-1279353 para o grupo vivo, $\mathrm{p}<0,05)$.

Da mesma forma, no subgrupo óbito encontramos níveis de óxido nítrico mais elevados em oposição ao subgrupo vivo (Figura 2, Mín 71,98 - Máx 182, Intervalo de confiança 95\% 86,44 - 143,3 para o grupo óbito e Min 
57,41- Máx 108,7, Intervalo de confiança 95\%

$76,73$ - 89,54 para o grupo vivo, $\mathrm{p}<0,05)$.

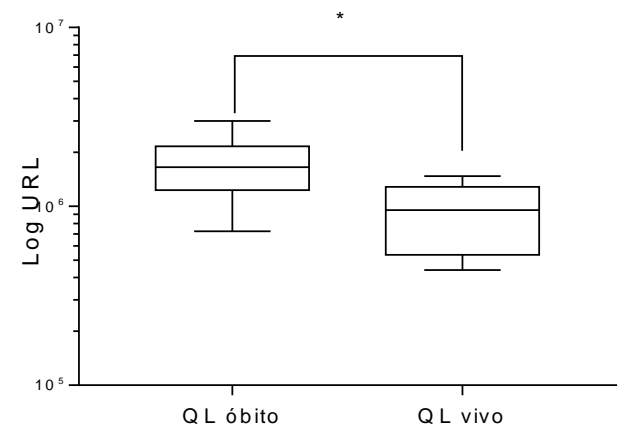

Figura 1 - Perfil de lipoperoxidação plasmática das pacientes portadoras de câncer de mama que foram a óbito em comparação com as pacientes vivas. Os dados representam a integral dos níveis médios de emissão medidos por quimioluminescência de alta sensibilidade induzida por tert-butil hidroperóxido. URL = unidades relativas de luz. $\mathrm{QL}=$ perfil de quimioluminescência. * indica $\mathrm{p}<0,05$, teste de Mann-Whitney.

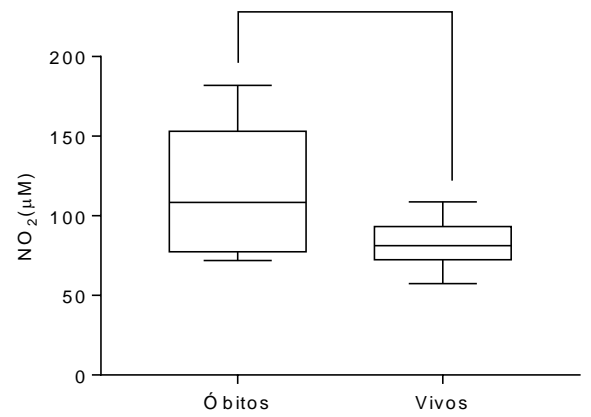

Figura 2 - Níveis de óxido nítrico plasmático das pacientes portadoras de câncer de mama que foram a óbito em comparação com as pacientes vivas. $\mathrm{NO}=$ óxido nítrico. * indica $\mathrm{p}<0,05$, teste de MannWhitney.

A presença de metástases no grupo vivo se correlacionou positivamente com o valor dos níveis de hidroperóxidos $(\mathrm{R}=0,7827 ; \mathrm{p}=0,0238)$ e com a presença de êmbolos angiolinfáticos ( $R$ $=0,5424 ; \mathrm{p}=0,0029)$. No grupo óbito, o grau histológico se correlacionou diretamente com o nível trófico adiposo $(\mathrm{R}=0,6587 ; \mathrm{p}=0,0323)$ e a idade ao diagnóstico foi inversa à concentração de NO $(R=-0,8571 ; p=0,0238)$.

\section{Discussão}

Neste estudo piloto demonstramos vez que pacientes com câncer de mama que tiveram o óbito como desfecho clínico apresentaram tanto o perfil de lipoperoxidação plasmática quanto os níveis de metabólitos do NO mais elevados ao diagnóstico, quando comparadas às pacientes vivas. Além disso, no grupo de pacientes vivas os valores de lipoperóxidos no momento do diagnóstico foram diretamente relacionados com o desenvolvimento de metástases, condição sabidamente associada ao pior prognóstico e ocorrência de morte no câncer de mama. Até onde sabemos, este é o primeiro estudo a demonstrar esta associação.

Sabe-se que a heterogeneidade do tumor determina que, embora certos tipos de câncer ou subpopulação de células se beneficiem de terapias baseadas em EROS, o estresse oxidativo pode potencialmente instigar efeitos indesejáveis em outras células, como o aumento da instabilidade genética e a ocorrência de mutações $^{9,16}$. Essa sequência dinâmica de 
eventos e a pressão constante para reajustes celulares pode acabar promovendo a evolução de células resistentes aos quimioterápicos. Além disso, estudos apontam que o estresse oxidativo é capaz de afetar uma subpopulação de células malignas residuais altamente resistentes ao tratamento, as chamadas células tronco cancerosas $^{17}$. Acredita-se que tais células sejam responsáveis pela recorrência do câncer após a quimioterapia ou radioterapia, e o excesso de EROS pode induzir a superexpressão de genes da via de sinalização Wnt / $\beta$-catenina, responsável por controlar a capacidade de autorenovação de células-tronco cancerosas em vários tipos de cânceres, incluindo o câncer de $\operatorname{mama}^{17,18}$.

Ao analisarmos o grupo vivo, a presença de metástases foi maior em pacientes com a existência de êmbolos angiolinfáticos. Sabe-se que os êmbolos angiolinfáticos propiciam um microambiente hipóxico, que influencia as células malignas impulsionando a progressão do tumor. A maior parte da resposta à hipóxia é desencadeada por fatores de transcrição denominados fatores induzíveis por hipóxia (HIFs), que induzem a expressão de genes que auxiliam as células a se adaptarem a ambientes com baixa concentração de $\mathrm{O}_{2}$. Dentre os HIFs, o papel do HIF-1 é o melhor estabelecido no avanço tumoral. Ele é o responsável por ativar as vias de sinalização associadas a transição epitélio mesenquimal, durante a qual células cancerígenas perdem o contato célula a célula, se desprendem do tumor primário e invadem o estoma adjacente, penetram na corrente sanguínea e se disseminam para sítios distantes formando tumores secundários ${ }^{19}$. Esse tem sido considerado o primeiro passo no processo de metástase ${ }^{20}$. Além disso, para a invasão tecidual, as células cancerígenas precisam degradar a membrana basal circundante, o que ocorre com o auxílio das chamadas metaloproteinases da matriz (MMPs), com destaque para a MPP-2 e MPP-9, que degradam o colágeno tipo IV, um importante componente da membrana basal. Muitos estudos sugerem que a hipóxia e o HIF1 regulam positivamente os níveis de expressão e a atividade das MPP-2 e MPP-9 $9^{21,22}$. O HIF-1 também é responsável por promover o extravasamento de células neoplásicas da circulação para um microambiente tecidual e regular a formação de um nicho pré-metastático em órgãos distantes, antes da chegada das células cancerígenas ${ }^{19,23}$. 
Quando estudamos a relação entre a idade ao diagnóstico e a concentração dos metabólitos do NO no subgrupo óbito obtivemos uma relação negativa, ou seja, quanto mais velhas as pacientes ao diagnóstico menores as concentrações obtidas. Diversos estudos apontam que mulheres na pós-menopausa apresentam tumores de mama biologicamente menos agressivos e de melhor prognóstico quando comparadas as mulheres jovens ${ }^{24,25}$. Sugere-se que isso ocorra em decorrência de, em pacientes mais jovens, uma doença mais avançada no momento do diagnóstico ou em razão de divergências na biologia tumoral, além da maior associação com a presença de história familiar e mutações genéticas nas mulheres mais $\operatorname{novas}^{26}$.

Em nosso estudo, o grau histológico se correlacionou com o nível trófico adiposo nas pacientes que foram a óbito. As evidências atuais sugerem que a inflamação do tecido adiposo é um ambiente favorável ao desenvolvimento do câncer ${ }^{27}$. Além disso, a desregulação na produção de fatores do tecido adiposo como citocinas inflamatórias, adipocinas e estrogênios gerados pela aromatização de andrógenos, podem afetar outros órgãos através de efeitos parácrinos e endócrinos, contribuindo para progressão tumoral $^{28}$. Sabemos que obesidade, inflamação crônica e câncer de mama estão intimamente relacionados, sendo recomendado que os médicos orientem seus pacientes quanto a intervenções dietéticas e de estilo de vida em pacientes com sobrepeso ou obesidade.

Dentre as limitações deste estudo incluem-se o tamanho da amostra do grupo óbito e a heterogeneidade intrínseca entre os grupos óbito e vivo.

\section{Conclusão}

Os dados aqui apresentados indicam que é possível distinguir o perfil oxidativo das pacientes ainda em diagnóstico, sendo uma medida indireta de um provável desfecho desfavorável em pacientes com câncer de mama em estadios operáveis. Dessa forma, é plausível que sejam adotadas terapias mais agressivas e/ou maior vigilância para mulheres com perfil de pior prognóstico, destacando a medida do perfil oxidativo como uma ferramenta para auxiliar a tomada de decisões pelos clínicos no futuro. 
Tabela 1 - Dados clinicopatológicos das pacientes estudadas.

\begin{tabular}{|c|c|c|c|c|}
\hline & Vivo & Óbito & & \\
\hline \multicolumn{5}{|l|}{ Idade ao diagnóstico } \\
\hline$\leq 50$ anos & $27.8 \%$ & $27.8 \%$ & $\mathrm{P}<0.05$ & \\
\hline$>50$ anos & $72.2 \%$ & $72.2 \%$ & $\mathrm{P}<0.05$ & \\
\hline \multicolumn{5}{|l|}{ Grau histológico } \\
\hline Grau I & $44.4 \%$ & $18.7 \%$ & $\mathrm{P}<0.05$ & \\
\hline Grau II & $41.7 \%$ & $50.0 \%$ & $\mathrm{P}<0.05$ & \\
\hline Grau III & $13.9 \%$ & $31.3 \%$ & $\mathrm{P}<0.05$ & \\
\hline \multicolumn{5}{|l|}{ Expressão RE/RP } \\
\hline RE/RP positivo + ki67<14\% & $30.6 \%$ & $5.9 \%$ & $\mathrm{P}<0.05$ & \\
\hline RE/RP positivo + ki67>14\% & $50.0 \%$ & $35.3 \%$ & $\mathrm{P}<0.05$ & \\
\hline RE/RP negativo e qualquer ki67\% & $19.4 \%$ & $58.8 \%$ & $\mathrm{P}<0.05$ & \\
\hline \multicolumn{5}{|l|}{ Metástase linfonodal } \\
\hline Não & $62.9 \%$ & $40.0 \%$ & $\mathrm{P}<0.05$ & \\
\hline Sim & $37.1 \%$ & $60.0 \%$ & $\mathrm{P}<0.05$ & \\
\hline \multicolumn{5}{|l|}{ Êmbolos angiolinfáticos } \\
\hline Não & $58.8 \%$ & $33.3 \%$ & $\mathrm{P}<0.05$ & \\
\hline Sim & $41.2 \%$ & $66.7 \%$ & $\mathrm{P}<0.05$ & \\
\hline \multicolumn{5}{|l|}{ Menopausa ao diagnóstico } \\
\hline Não & $27.8 \%$ & $27.8 \%$ & $\mathrm{P}<0.05$ & \multirow{13}{*}{$\begin{array}{l}\text { Legenda: } \mathrm{RE} \\
=\text { receptor } \mathrm{de} \\
\text { estrogênio. } \mathrm{RP} \\
=\text { receptor } \mathrm{de} \\
\text { progesterona. }\end{array}$} \\
\hline Sim & $72.2 \%$ & $72.2 \%$ & $\mathrm{P}<0.05$ & \\
\hline Tamanho do tumor & & & & \\
\hline$\leq 2 \mathrm{~cm}$ & $40.0 \%$ & $41.2 \%$ & $\mathrm{P}<0.05$ & \\
\hline Entre 2 e $5 \mathrm{~cm}$ & $48.6 \%$ & $23.5 \%$ & $\mathrm{P}<0.05$ & \\
\hline$\geq 5 \mathrm{~cm}$ & $11.4 \%$ & $35.3 \%$ & $\mathrm{P}<0.05$ & \\
\hline \multicolumn{4}{|l|}{ Ki-67 } & \\
\hline$<14 \%$ & $44.4 \%$ & $31.3 \%$ & $\mathrm{P}<0.05$ & \\
\hline$\geq 14 \%$ & $66.6 \%$ & $68.7 \%$ & $\mathrm{P}<0.05$ & \\
\hline \multicolumn{4}{|l|}{ Índice de massa corporal $\left(\mathrm{kg} / \mathrm{m}^{2}\right)$} & \\
\hline Eutrófica & $37.1 \%$ & $38.9 \%$ & $\mathrm{P}<0.05$ & \\
\hline Sobrepeso & $45.7 \%$ & $38.9 \%$ & $\mathrm{P}<0.05$ & \\
\hline Obesa & $17.2 \%$ & $22.2 \%$ & $\mathrm{P}<0.05$ & \\
\hline
\end{tabular}


5. Mencalha A, Victorino VJ, Cecchini R,

\section{Referências}

1. Halliwell B, Gutteridge JMC. Free radicals in biology and medicine. New York, Oxford University, 4th edition, 2007.

2. Pani G,

Giannoni E, Galeotti T, Chiarugi P.

Redox-based escape mechanism from death: the cancer lesson. Antiox Redox Signal 11(11): 2791-2802, 2009.

3. Panis, C., Victorino, V. J., Herrera, A. C., Cecchini, A. L., Simão, A. N., Tomita, L. Y., \& Cecchini, R. Can Breast Tumors Affect the Oxidative Status of the Surrounding Environment? A Comparative Analysis among Cancerous Breast, Mammary Adjacent Tissue, and Plasma. Oxid Med Cell Longev. 2015;2015:6429812.

4. Shaw PG, Chaerkady R, Wang T, Vasilatos S, Huang Y, van

Houten B, Pandey A, Davidson NE.

Integrated proteomic and metabolic analysis of breast cancer progression. PLoS

ONE 8(9): e76220, 2013.
Panis C. Mapping oxidative changes in breast cancer: understanding the basic to reach the clinics. Anticancer Res 34: 1127-1140, 2014.

6. Gauthier N, Arnould L, Chantôme A, R eisser D, Bettaieb A, Reveneau S, Jean nin JF. To stimulate or to inhibit nitric oxide production in mammary tumors? Bull Cancer 91(9): 705$712,2004$.

7. Fang J, Nakamura H, Iyer AK. Tumortargeted induction of oxystress for cancer therapy. J Drug Target 15(78): 475-486, 2007.

8. Pires BRB, Panis C, Alves VD, Herrera ACSA, Binato R, Pizzatti L, Cecchini R, Abdelhay E. Label-Free Proteomics Revealed Oxidative Stress and Inflammation as Factors That Enhance Chemoresistance in Luminal Breast Cancer. Oxid Med Cell Longev. 2019;2019:5357649.

9. de Sa Junior PL, Camara DAD, Porcacchia AS, Fonseca PMM, Jorge SD, Araldi RP, Ferreira AK. The roles of ROS in cancer heterogeneity and 
therapy. Oxid Med Cell Longev. 2017;2017:2467940.

10. Herrera AC, Victorino VJ, Campos FC, Verenitach BD, Lemos LT, Aranome AM, Oliveira SR, Cecchini AL, Simão AN, Abdelhay E, Panis C, Cecchini R. Impact of tumor removal on the systemic oxidative profile of patients with breast cancer discloses lipid peroxidation at diagnosis as a putative marker of disease recurrence. Clin Breast Cancer. 2014;14:451-9.

11. Lemos LG, Victorino VJ, Herrera AC, Aranome AM, Cecchini AL, Simão AN, Panis C, Cecchini R. Trastuzumabbased chemotherapy modulates systemic redox homeostasis in women with HER2-positive breast cancer. Int Immunopharmacol. 2015 Jul;27(1):814.

12. Victorino VJ, Campos FC, Herrera AC, Colado Simão AN, Cecchini AL, Panis C, Cecchini R. Overexpression of HER$2 /$ neu protein attenuates the oxidative systemic profile in women diagnosed with breast cancer. Tumour Biol. 2014 Apr;35(4):3025-34.
13. Panis C, Victorino VJ, Herrera AC, Freitas LF, De Rossi T, Campos FC, Simão AN, Barbosa DS, Pinge-Filho P, Cecchini R, Cecchini AL. Differential oxidative status and immune characterization of the early and advanced stages of human breast cancer.

Breast Cancer Res Treat. 2012 Jun;133(3):881-8.

14. Sedlak, J, Lindsay RH. Estimation of total, protein-bound, and nonprotein sulfhydryl groups in tissue with Ellman's reagent. Anal. Biochem 1968, 25: 192-205

15. Gonzales-Flecha B, Lleswy S, Boveris A. Hydroperoxide-initiated chemiluminescence: An assay for oxidative stress in biopsies of heart, liver and muscle. Free Rad Biol Med 1991, 10: 93-100.

16. Okon IS, Zou MH. Mitochondrial ROS and cancer drug resistance: Implications for therapy. Pharmacol Res. 2015;100:170-174.

17. Dayem AA, Choi HY, Kim JH, Cho SG. Role of oxidative stress in stem, cancer, and cancer stem cells. Cancers (Basel). 2010;2(2):859-884. 
18. Waris G, Ahsan H. Reactive oxygen species: role in the development of cancer and various chronic conditions. J Carcinog. 2006 May 11;5:14.

19. Liu ZJ, Semenza GL, Zhang HF. Hypoxia-inducible factor 1 and breast cancer metastasis. J Zhejiang Univ Sci B. $2015 ; 16(1): 32-43$.

20. Chaffer CL, Weinberg RA. A perspective on cancer cell metastasis. Science. 2011;331(6024):15 59-1564.

21. Krishnamachary B, Zagzag D, Nagasawa H, Rainey K, Okuyama H, Baek JH, Semenza GL. Hypoxiainducible factor-1-dependent repression of E-cadherin in von Hippel-Lindau tumor suppressor-null renal cell carcinoma mediated by TCF3, ZFHX1A, and ZFHX1B. Cancer Res. 2006 Mar 1;66(5):2725-31.

22. Muñoz-Nájar UM, Neurath KM, Vumbaca F, Claffey KP. Hypoxia stimulates breast carcinoma cell invasion through MT1-MMP and MMP2 activation. Oncogene. 2006 Apr 13;25(16):2379-92.
23. Wong CC, Gilkes DM, Zhang H, Chen J, Wei H, Chaturvedi P, Fraley SI, Wong CM, Khoo US, Ng IO, Wirtz D, Semenza GL. Hypoxia-inducible factor 1 is a master regulator of breast cancer metastatic niche formation. Proc Natl Acad Sci U S A. 2011 Sep 27;108(39):16369-74.

24. Dutra MC, Rezende MA, Andrade VP, Soares FA, Ribeiro MV, Paula EC, \& Gobbi H. Imunofenótipo e evolução do câncer de mama: comparação entre mulheres muito jovens e mulheres na pós-menopausa. Rev Bras Ginecol e Obstet. 2009; 31(2):54-60

25. Sundquist M, Thorstenson S, Brudin L, Wingren S, Nordenskjöld B. Incidence and prognosis in early onset breast cancer. Breast. 2002; 11(1):30-5.

26. Chan A, Pintilie M, Vallis K, Girourd C, Goss P. Breast cancer in women $<$ or $=$ 35 years: review of 1002 cases from a single institution. Ann Oncol. 2000; 11(10):1255-62.

27. Goodwin PJ, Stambolic V. Impact of the obesity epidemic on cancer. Annu Rev Med. 2015;66(1):281-296. 
28. Kang C, LeRoith D, Gallagher EJ.

Diabetes, Obesity, and Breast

Cancer. Endocrinology.

2018;159(11):3801-3812.

Agradecimentos - Agradecimentos ao

Laboratório de Biologia de Tumores e ao

Hospital do Câncer de Francisco Beltrão pelo suporte técnico e possibilidade de realização da presente pesquisa.

Financiamento - Fundação Araucária, Conselho Nacional de Desenvolvimento Tecnológico e Programa de Apoio à Pesquisa no SUS.

Reservado aos Editores

Data de submissão: 17/05/2021

Data de aprovação: 09/06/2021 